\title{
Evaluation of Combinatorial Capacity of Coconut and Cocoa Plant Growth Promoting Rhizobacteria (PGPR) with Biocontrol Agent Trichoderma harzianum
}

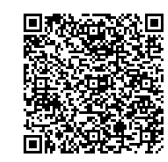

\author{
Khadeejath Rajeela TH, Alka Gupta*, Murali Gopal, Vinayaka Hegde and George V Thomas \\ ICAR-Central Plantation Crops Research Institute, India
}

Received: 盋June 19,2018; Published: 阱July 06, 2018

*Corresponding author: Alka Gupta, ICAR-Central Plantation Crops Research Institute, Kudlu PO, Kasaragod, Kerala-671124, India

\begin{abstract}
Combining PGPR and biocontrol agents that are compatible with each other is a strategic approach to enhance plant growth and development, control plant diseases and pests. A screening study was carried out to evaluate the compatibility of eight PGPR isolated from the rhizosphere and endorhizosphere of coconut (Pseudomonas putida KnSF208, Bacillus licheniformis RSB14, Bacillus megaterium TEB2, Bacillus megaterium TSB16) and cocoa (Bacillus cereus ASB3, Bacillus subtilis VEB4, Bacillus licheniformis KGEB16, Pseudomonas putida KDSF23) with fungal antagonist, Trichoderma harzianum, under in vitro conditions. The Trichoderma harzianum was isolated from the rhizosphere of coconut. All the PGPR isolates were tested for compatibility with Trichoderma harzianum by dual culture technique on four different media. Among the coconut PGPR isolates tested, Bacillus megaterium TSB16 was found to be compatible with Trichoderma harzianum on nutrient agar and King's B agar. However, none of the cocoa PGPR were compatible with Trichoderma harzianum on all the media tested. This study indicates that combined application of PGPR Bacillus megaterium TSB16 and Trichoderma harzianum, as bioinoculants, is possible for dual benefits of enhanced plant growth and soilborne pathogen suppression.
\end{abstract}

Keywords: Bacillus megaterium; Trichoderma harzianum; Compatibility; Fluorescent Pseudomonas

\section{Introduction}

Beneficial rhizosphere organisms are generally classified into two broad groups based on their primary beneficial effect on plant growth: (a) microorganisms with direct effects on plant growth promotion and (b) biological control agents that indirectly assist with plant productivity through the control of plant pathogens. Co-inoculation of plant growth promoting rhizobacteria (PGPR) and bio control agents (BCAs) is considered to be an innovative approach in plant-health management, and for the improvement of crop yield and quality. The use of formulated preparations, consisting of a single microbial species or strains as inoculants has often resulted in inconsistent performances in agriculture [1]. One of the reasons of such a failure could be that a single strain might not grow equally well in a variety of environmental conditions [2]. Thus, more emphasis was laid on the combined use of beneficial microorganisms as they will have the advantage of exercising a broad-spectrum activity, more stable rhizosphere community, enhancing the efficacy and reliability of biological control generally and ensuring greater induction of defense enzymes over individual strains [3].
Application of binary or multiple mixtures would mimic the natural situation more closely and might broaden the spectrum of biocontrol activity [1]. Combining such beneficial organisms can enhance the plant's innate resistance level against the invading pathogens more than their individual effort. In particular, combinations of fungi and bacteria may provide protection at different times or under different conditions and occupy different or complementary niches [4]. Such combinations may overcome inconsistencies in the performance of individual isolates. It was reported that the consortia of Trichoderma harzianum, fluorescent Pseudomonas and Glomus intraradices against Fusarium wilt not only suppressed the disease incidence but also helped in sustenance and growth promotion of crop through their different plant growth enhancement and nutrient uptake properties [5]. Interestingly, several researchers have observed increased plant growth and improved disease control using microbial consortia comprising of various biocontrol organisms such as Trichoderma, Pseudomonas, Bacillus spp., etc. in wheat, radish, chickpea, tomato, pepper, Arabidopsis and pigeon pea [5]. 
Rhizosphere facilitates growth, development and functioning of diverse microbial communities including plant growth-promoting rhizobacteria (PGPR). PGPR colonize the root surfaces, promote plant growth and protect plants from phytoparasites [6]. The rhizosphere is a nutrient-rich habitat influenced by the chemical and biological processes of root, which is an ideal place for the proliferation of these microbes [7,8]. PGPR may promote plant growth by several mechanisms which entail nitrogen fixation, sequestration of iron for plants by siderophores, production of plant hormones like auxins, cytokinins and gibberellins and lowering of plant ethylene levels [9]. PGPR have the potential capability to significantly enhance the yields of various crops [10]. Trichoderma species are plant symbionts that live free in the rhizosphere [11]. The soil fungus Trichoderma harzianum is used as biocontrol agent using its antagonistic abilities against phytopathogenic fungi, although it also has direct effects on plants, increasing or accelerating their growth and resistance to diseases and tolerance to abiotic stresses.

Biocontrol by Trichoderma is achieved through several mechanisms with a combination of two or more mechanisms acting together, probably responsible for the versatility of its biocontrol. A well-known mycoparasite, it secretes cell wall-degrading enzymes and other compounds that can directly kill the target pathogen. A competent rhizosphere colonizer, it can compete for space and nutrients with other microorganisms in the rhizosphere. Depending upon the strains, the use of Trichoderma species in agriculture can provide numerous advantages viz. rhizosphere competence allowing the strains to establish rapidly within the stable microbial communities in the rhizosphere; control of pathogenic and competitive or deleterious microflora by using a variety of mechanisms; improvement of the plant health and stimulation of root growth [12]. So far, Trichoderma species are among the most studied fungal biocontrol agents and commercially marketed as biopesticides, biofertilizers and soil amendments [13]. Compatibility and effectiveness of combinations of Trichoderma with other beneficial organisms is an important issue [14]. Therefore, the present study was undertaken to investigate the compatibility of eight PGPR, isolated from the rhizosphere and roots of coconut and cocoa, to fungal antagonist Trichoderma harzianum.

\section{Materials and Methods}

\section{Cultures}

Trichoderma harzianum culture was obtained from the Crop Protection Division, Central Plantation Crops Research Institute Kasaragod, which was previously isolated from the rhizosphere of coconut [15]. Fungal cultures were maintained on Potato Dextrose Agar (PDA) slants from which fresh cultures were prepared for further use.

\section{PGPR strains}

The compatibility of eight PGPR was tested against the biocontrol agent Trichoderma harzianum. Of the eight PGPR, four
(Pseudomonas putida KnSF208, Bacillus licheniformis RSB14, Bacillus megaterium TEB2 and Bacillus megaterium TSB16) were isolated from the rhizosphere and endorhizosphere of coconut and the other four (Bacillus cereus ASB3, Bacillus subtilis VEB4, Bacillus licheniformis KGEB16 and Pseudomonas putida KDSF23) were isolated from the rhizosphere and endorhizosphere of cocoa. These PGPR were selected based on their plant growth promoting characteristics, performance based on seedlings study, green house experiments and field trials in coconut and cocoa $[16,17]$. The isolates were maintained on the nutrient agar slants at $4{ }^{\circ} \mathrm{C}$ for further use.

\section{Selection of suitable medium for antagonistic studies}

Eight selected Bacillus species and Pseudomonas species along with T. harzianum were inoculated on different media like Potato Dextrose Agar (PDA), Sabouraud Dextrose Agar (SDA), Nutrient Agar (NA) and King's B Agar (KBA) to select an appropriate medium which would allow both bacteria and fungus to grow well for compatibility studies.

\section{Compatibility study}

Dual culture technique was performed for evaluating the compatibility of PGPR with Trichoderma harzianum in different media viz. NA and KBA. Fungal cultures, grown on PDA plates at $30^{\circ} \mathrm{C}$ for 3 to 4 days, were used for the study. Bacillus and Pseudomonas species were raised in nutrient broth and King's B broth, respectively. Twenty four hr. old bacterial cultures were streaked at four equidistant points along the periphery of the NA and KBA plates. Mycelial discs of Trichoderma harzianum were cut out from the edge of an actively growing colony with the help of a sterile $5 \mathrm{~mm}$ diameter cork borer and placed upside down at the centre of the assay plates. Control plate was kept without bacterial inoculation. All the plates were incubated at $30^{\circ} \mathrm{C}$. When the hyphal growth of Trichoderma in the control plates reached the periphery, the growth of fungus in the dual inoculation plates (Trichoderma harzianum+PGPR) were measured for assessing compatibility. The zone of inhibition was measured and percent inhibition over control was calculated using the formula R1-R2/R1x100 where, $\mathrm{R} 1$ is maximum radius of mycelial growth on the control plate and $\mathrm{R} 2$ is radius of mycelial growth directly opposite to the bacterial growth [18].

\section{Results}

Out of four media tested, PDA and SDA, though favored the growth of Trichoderma harzianum but did not suit the growth of all the bacterial isolates. Hence, they were not suitable for studying compatibility. The in vitro compatibility studies were, therefore, carried out using NA and KBA media, which supported growth of both Trichoderma harzianum and PGPR bacteria. Strains of Pseudomonas putida (KnSF208), Bacillus megaterium (TSB16 and TEB2) and Bacillus licheniformis (RSB14) isolated from coconut rhizosphere and endorhizosphere were tested in vitro for compatibility. Bacillus megaterium TSB16 overgrew Trichoderma harzianum on all the 
media tested, no inhibition zone formed between these two isolates indicating that these organisms were compatible. Coconut isolate Bacillus megaterium TEB2 was compatible with Trichoderma harzianum on KBA medium (Figure 1). The isolate showed $40 \%$ inhibition when co-cultured on nutrient agar. Among the PGPR tested, Pseudomonas putida KnSF208 showed mycelia inhibition of $40 \%$ on NA and $37 \%$ on KBA (Table 1), (Figure1). The coconut isolate Bacillus licheniformis RSB14 showed highest inhibition of $44 \%$ on nutrient agar.
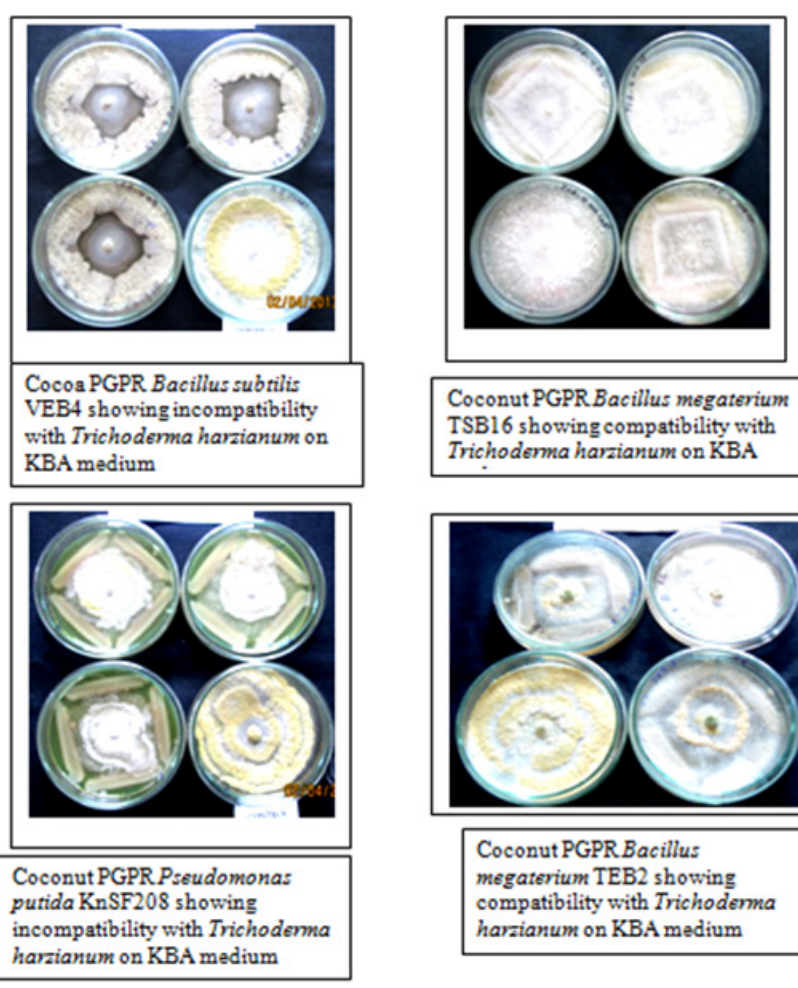
TSB16 showing compatibility with Trichodema harzianum on KBA

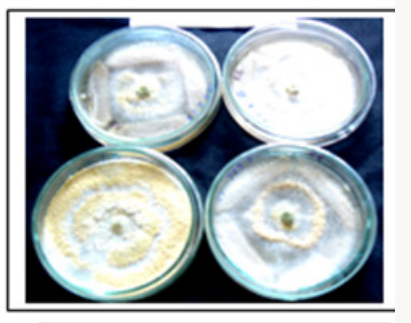

Coconut PGPR Bacillus megaterium TEB2 showing compatibility with Trichodenma harsianum on KBA medium

Figure1: PGPR isolates showing antagonism against Trichoderma harzianum.

Table 1: Compatibility of Coconut PGPR on Different Media.

\begin{tabular}{|c|c|c|}
\hline \multirow{2}{*}{ Isolate ID } & \multicolumn{2}{|c|}{$\begin{array}{c}\text { \% Inhibition of Trichoderma } \\
\text { harzianum }\end{array}$} \\
\cline { 2 - 3 } & Nutrient agar & King's B agar \\
\hline Bacillus megaterium TSB16 & 0 & 0 \\
\hline Bacillus megaterium TEB2 & $40 \pm 4.49$ & 0 \\
\hline $\begin{array}{c}\text { Pseudomonas putida } \\
\text { KnSF208 }\end{array}$ & $40 \pm 2.34$ & $37 \pm 1.68$ \\
\hline Bacillus licheniformis RSB14 & $44 \pm 2.96$ & $40 \pm 1.72$ \\
\hline
\end{tabular}

All the PGPR isolated from cocoa rhizosphere and endorhizosphere were incompatible with Trichoderma harzianum on NA and KBA. Of the cocoa PGPR tested, Bacillus cereus ASB3 showed maximum inhibition of mycelia growth of Trichoderma harzianum (65\%) on nutrient agar. This was followed by Bacillus subtilis VEB4 which recorded 60\% inhibition of Trichoderma over control on King's B agar (Figure 1). The cocoa isolate Pseudomonas putida KDSF23 recorded the least mycelial growth inhibition of
$23 \%$ on King's B agar and $30 \%$ on nutrient agar (Table 2). As far as the medium used was concerned, maximum inhibition of hyphal growth of Trichoderma harzianum was obtained on nutrient agar as compared to King's B agar.

Table 2: Compatibility of Cocoa PGPR on Different Media.

\begin{tabular}{|c|c|c|}
\hline \multirow{2}{*}{ Isolate ID } & \multicolumn{2}{|c|}{ \% Inhibition of Trichoderma harzianum } \\
\cline { 2 - 3 } & Nutrient agar & King's B agar \\
\hline Bacillus cereus ASB3 & $65 \pm 2.28$ & $54 \pm 2.54$ \\
\hline $\begin{array}{c}\text { Pseudomonas putida } \\
\text { KDSF23 }\end{array}$ & $30 \pm 3.41$ & $23 \pm 5.91$ \\
\hline $\begin{array}{c}\text { Bacillus licheniformis } \\
\text { KGEB16 }\end{array}$ & $52 \pm 2.35$ & $45 \pm 0.63$ \\
\hline Bacillus subtilis VEB4 & $49 \pm 2.34$ & $60 \pm 3.41$ \\
\hline
\end{tabular}

\section{Discussion}

The PGPR isolated from coconut and cocoa rhizosphere and roots were individually screened for their compatibility with Trichoderma harzianum in dual culture test. For carrying out compatibility studies, both NA and KBA were found suitable and PGPR and the fungal biocontrol agent exhibited satisfactory growth on these media. However, all the tested isolates showed maximum inhibition of growth of Trichoderma harzianum on nutrient agar medium. Greater levels of antagonism on the nutrient agar medium could be related to more suitable conditions for synthesis of antagonistic bioactive molecules. Peptone had been reported as a key nutrient for the production of antifungal compounds by Bacillus amyloliquefaciens RC-2 [19]. Also, variation in the antagonism of the native strains against fungi was observed on different solid media [20]. Antagonistic properties of Pseudomonas species were also reported to be influenced by culture medium composition, the fungal pathogen, and its growth stages [21]. Coconut isolate, Bacillus megaterium TSB16, was found to be compatible with Trichoderma harzianum on both media tested.

A positive interaction existed between Bacillus megaterium TSB16 and the fungal antagonist, Trichoderma harzianum. It could be attributed to the existence of synergism between the metabolites produced by PGPR and Trichoderma harzianum. Our findings corroborate the report of [22]. who found that the rhizobacterial strains, Bacillus subtilis, Bacillus pumilus and Bacillus cereus did not inhibit growth of Trichoderma harzianum in in vitro assays. Combinations of fungi and bacteria might provide protection at different times or under different conditions and might probably mimic the natural situation in the rhizosphere [23]. Bacillus licheniformis RSB14 isolated from the rhizosphere of coconut inhibited the mycelial growth of Trichoderma harzianum by an inhibition per cent of $44 \%$. It had been earlier reported that the Bacillus licheniformis RSB14 had antagonistic activity against Ganoderma applanatum (57\%) and Thielaviopsis paradoxa (80\%) in in vitro studies George and workers [24] had also reported that the Bacillus licheniformis RSB14 had the potential to produce 
siderophore, chitinase, ammonia, $\beta$-1, 3- glucanase and salicyclic acid.

Chitinases are the cell wall-degrading enzymes that degrade chitin, a common constituent of fungal cell walls that is made up of $\beta$-1, 4-linked homopolymers of $\mathrm{N}$-acetylglucosamine [25]. The antifungal metabolites such as $\beta$-1,3-glucanase and $\beta$-1,4-glucanase degrade the components of fungal cell wall such as chitin, $\beta-1,3-$ glucan and glucosidic bonds [26]. Therefore, it was likely that cell wall lysis would have been due to concerted action of chitinase and $\beta$-1,3-glucanase. Generally, Bacillus species are capable of producing variety of fungal cell wall-degrading enzymes, such as chitinase, proteinase, cellulase and amylase [27]. Production of chitinase, $\beta$-1,3-glucanase, ammonia and siderophore by Bacillus licheniformis RSB14 might have collectively contributed to inhibition of fungal growth. Ghasemi [28] reported that halotolerant bacterium, Bacillus pumilus strain SG2 produced chitinases which had antifungal activity against Rhizoctonia solani, Verticillium species, etc. It was also reported that Bacillus megaterium and Bacillus subtilis inhibited the growth of Aspergillus niger in plate assay by the production of antifungal substances such as chitinase, cellulase and protease [29].

Pseudomonas putida KDSF23 isolated from cocoa and Pseudomonas putida KnSF208 isolated from coconut were also found to inhibit Trichoderma harzianum. The strains had the potential to produce siderophores $[16,17]$. Competition for iron by siderophore production had been considered as one of the important mechanisms by which fluorescent pseudomonads exert their antagonistic activity and plant growth promotion. Siderophores produced by the microorganisms could bind iron with high specificity and affinity, making the iron unavailable for other microorganisms, and thereby limiting their growth. Siderophores might play an important role in the competition between microorganisms and may also act as growth promoters [30]. In an earlier report, Pseudomonas aeruginosa showed strong antagonism against two fungal pathogens, Macrophomina phaseolina and Fusarium oxysporum through the production of siderophores and HCN [[26]. Costa and coworkers [31] found that most of the Pseudomonas species displaying antifungal activity were siderophore producers.

All the cocoa isolates tested were found to be incompatible to Trichoderma harzianum and per cent inhibition ranged from $23 \%$ to $64 \%$ (Table 2). Among the cocoa isolates, the maximum inhibition of Trichoderma harzianum was shown by Bacillus cereus ASB3, isolated from cocoa rhizosphere, and Bacillus subtilis VEB4, isolated from the endorhizosphere of cocoa. A plausible reason for their antagonistic effect could be the production of secondary metabolites, such as antibiotics which resulted in inhibition of mycelial growth of Trichoderma harzianum. Antibiotics are lowmolecular weight compounds produced by microorganisms that are deleterious to the metabolism or growth of other microorganisms. It is well known that most of the Bacillus strains, such as Bacillus subtilis and Bacillus cereus, produce antibiotics such as d-gluconic acid and 2-hexyl-5-propyl resorcinol and bioactive compounds belonging to the cyclic lipopeptides [6]. In addition Bacillus subtilis VEB4 was found to be an antagonist to Phytophthora palmivora in an earlier study and the percent inhibition recorded was $45 \%$ over control [17].

Further, the strain was observed to produce siderophore, antibiotic and ammonia [17]. This suggested that the fungal mycelia inhibition happened not only by antibiosis but also by other antifungal metabolites such as siderophores, and gaseous product like ammonia. The cocoa isolates Pseudomonas putida KDSF23, Bacillus cereus ASB3 and Bacillus licheniformis KGEB16 had the potential to produce chitinases which also might have helped them to inhibit the growth of Trichoderma harzianum. Species of Pseudomonas excrete chitinases and $\beta$-1, 3-glucanases to digest the fungal cell wall chitin and glucan, respectively, and use these as a carbon and energy source [32]. Mostly Bacillus species were selected to play an important role in Trichoderma species inhibition [33]. Similar to our findings, Bacillus subtilis and Bacillus atrophaeus were reported to be inhibitory to Trichoderma harzianum in dual culture studies and were found to inhibit rhizome rot pathogens [22].

\section{Conclusion}

Out of eight PGPR, four from coconut and four from cocoa, tested for compatibility to Trichoderma harzianum, Bacillus megaterium TSB16 isolated from the rhizosphere of coconut was found to be compatible with Trichoderma harzianum. Among the four media tested, nutrient agar and King's B agar were observed to support the growth of both the fungal antagonist Trichoderma harzianum and PGPR. The results of this study permit the integration of fungal antagonist and PGPR for effective rhizosphere management in future.

\section{Acknowledgement}

We acknowledge the financial assistance provided by ICAR under the Network Project on "Application of Microorganisms in Agriculture and Allied Sectors" coordinated by National Bureau of Agriculturally Important Microorganisms, Mau, UP State, for carrying out this study. Khadeejath Rajeela is grateful for Senior Research Fellowship.

\section{References}

1. Raupach GS, Kloepper JW (1998) Mixture of plant growth promoting rhizobacteria enhance biological control of multiple cucumber pathogens. Phytopathology 88(11): 1158-1164.

2. Fukui R, Poinar EI, Bauer PH, Schroth MN, Hendson M, et al. (1994) Spatial colonization patterns and interaction of bacteria on inoculated sugar beet seed. Phytopathology 84: 1338-1345. 
3. Latha P, Anand T, Prakasam V, Jonathan EI, Paramathma M, Samiyappan R (2011) Combining Pseudomonas, Bacillus and Trichoderma strains with organic amendments and micronutrient to enhance suppression of collar and root rot disease in physic nut. Applied Soil Ecology 49: 215223.

4. Larkin RP, Fravel DR (1998) Efficacy of various fungal and bacterial organisms for control of Fusarium wilt of tomato. Plant Disease 82: 1022-1028.

5. Srivastava R, Khalid A, Singh US, Sharma AK (2010) Evaluation of arbuscular mycorrhizal fungus, fluorescent Pseudomonas and Trichoderma harzianum formulation against Fusarium oxysporum f. sp. lycopersici for the management of tomato wilt. Biological Control 53(1): 24-31.

6. Lugtenberg B, Kamilova F (2009) Plant-Growth-Promoting Rhizobacteria. Annu Rev Microbiol 63: 541-556.

7. Bakker PAHM, Berendsen RL, Doornbos RF, Wintermans PCA, Pieterse CMJ (2013) The rhizosphere revisited: root microbiomics. Frontiers in Plant Science 4 (165): 1-7.

8. Berendsen RL, Pieterse CM, Bakker PAHM (2012) The rhizosphere microbiome and plant health. Trends in Plant Science 17(8): 478-486.

9. Gupta Alka, Gopal Murali, Tilak KVBR (2000) Mechanism of plant growth promotion by rhizobacteria. Indian Journal of Experimental Biology 38: 856-862.

10. Glick BR, Cheng Z, Czarny J, Duan J (2007) Promotion of plant growth by ACC deaminase-producing soil bacteria. Eur J Plant Pathol 119(3): 329-339.

11. Alizadeh H, Behboudi K, Ahmadzadeh M, Javan Nikkhah M, Zamioudis $\mathrm{C}$ et al. (2013) Induced systemic resistance in cucumber and Arabidopsis thaliana by the combination of Trichoderma harzianum Tr6 and Pseudomonas sp. Ps14. Biological Control 65(1): 14-23.

12. Vinalea Francesco, Sivasithamparam K, Ghisalbertic EL, Marraa R, Wooa SL, Loritoa M, (2008) Trichoderma-plant-pathogen interactions. Soil Biology \& Biochemistry 40(1): 1-10.

13. Moran Diez, E Rubio B, Dominguez S, Hermosa R, Monte E, et al. (2012) Transcriptomic response of Arabidopsis thaliana after $24 \mathrm{~h}$ incubation with the biocontrol fungus Trichoderma harzianum. Journal of Plant Physiology 169(6): 614- 620.

14. Lorito M, Woo SL, Harman GE, Monte E (2010) Translational research on Trichoderma: from 'omics to the field. Annual Review of Phytopathology 48: 395-417.

15. Chandra Mohanan R, Prabha K Peter, Sharadraj KM (2013) Production technology of coir pith cake formulation of Trichoderma harzianum. Journal of Plantation Crops 41(2): 214-218.

16. George P (2013) Studies on Plant Growth Promoting Rhizobacteria mediated growth promotion in Coconut (Cocos nucifera L.) Thesis, Mangalore University, Karnataka, India, 234 p.

17. Thomas L (2013) Identification and evaluation of plant growth promoting rhizobacteria from the diverse bacilli and fluorescent pseudomonad population in rhizosphere and roots of cocoa (Theobroma cacao L.). Ph.D. Thesis, Mangalore University, Karnataka, India, 193 p.

18. Fernando, WGD, Pierson LS III (1999). The effect of increased phenazine antibiotic production on the inhibition of economically important soil-borne plant pathogens by Pseudomonas aureofaciens 30-84. Arch. Phytopath Plant Protec 32(6): 491-502.

19. Yoshida S, Hiradate S, Tsukamoto T, Hatakeda K, Shirata A (2001)
Antimicrobial activity of culture filtrate of Bacillus amyloliquefaciens RC-2 isolated from mulberry leaves. Phytopathology 91(2): 181-187.

20. Cordero P, Cavigliasso A, Principe A, Godino A, Jofre E, et al. (2012) Genetic diversity and antifungal activity of native Pseudomonas isolated from maize plants grown in a central region of Argentina. Systematic and Applied Microbiology 35(5): 342- 351.

21. Hammami I, Hsouna AB, Hamdi N, Gdoura R, Triki MA (2013) Isolation and characterization of rhizosphere bacteria for the biocontrol of the damping-off disease of tomatoes in Tunisia. CR Biologies 336(11-12): 557-564.

22. Shanmugam V, Gupta S, Dohroo NP (2013) Selection of a compatible biocontrol strain mixture based on co-cultivation to control rhizome rot of ginger. Crop Protection 43: 119-127.

23. Whipps JM (2001) Microbial interactions and biocontrol in the rhizosphere. J Exp Bot 52(1): 487-511.

24. George P, Gupta A, Gopal M, Chandra Mohanan R, Thomas L, et al. (2011) Antagonistic activity of coconut rhizospheric and endophytic Bacillus spp. against Ganoderma applanatum and Thielaviopsis paradoxa. Journal of Plantation Crops 39 (2): 278-284.

25. Sharma V, Shanmugam V (2011) Purification and characterization of a $24 \mathrm{kDa}$ chitobiosidase from the mycoparasitic fungus Trichoderma saturnisporum. J Basic Microbiol 52(3): 340-349.

26. Kumar S, Pandey P, Maheshwari DK (2009) Reduction in dose of chemical fertilizers and growth enhancement of sesame (Sesamum indicum L.) with application of rhizospheric competent Pseudomonas aeruginosa LES4. European Journal of Soil Biology 45: 334-340.

27. Adesina MF, Lembke A, Costa R, Speksnijder A, Smalla K (2007) Screening of bacterial isolates from various European soils for in vitro antagonistic activity towards Rhizoctonia solani and Fusarium oxysporum: site-dependent composition and diversity revealed. Soil Biology and Biochemistry 39(11): 2818-2828.

28. Ghasemi S, Ahmadian G, Jelodar NB, Rahimian H, Ghandili S, et al. (2010) Antifungal chitinases from Bacillus pumilus SG2: preliminary report World J Microbiol Biotechnol 26(8): 1437-1443.

29. Watcharin Yuttavanichakul, Pruksa Lawongsa, Sopone Wongkaew, Neung Teaumroong, Nantakorn Boonkerd, et al. (2012) Improvement of peanut rhizobial inoculant by incorporation of plant growth promoting rhizobacteria (PGPR) as biocontrol against the seed borne fungus, Aspergillus niger. Biological Control 63(2): 87-97.

30. Yu X, Ai C, Xin L, Zhou G (2011) The siderophore-producing bacterium, Bacillus subtilis CAS15, has a biocontrol effect on Fusarium wilt and promotes the growth of pepper. European Journal of Soil Biology 47(2): $138-145$.

31. Costa R, Gomes N, Peixoto RS, Rumjanekc N, Berg G, et al. (2006) Diversity and antagonistic potential of Pseudomonas spp. associated to the rhizosphere of maize grown in a subtropical organic farm. Soil Biol. Biochem 38(8): 2434 -2447.

32. Guinazu LB, Andres JA, Rovera M, Balzarini M, Rosas SB (2013) Evaluation of rhizobacterial isolates from Argentina, Uruguay and Chile for plant growth-promoting characteristics and antagonistic activity towards Rhizoctonia sp. and Macrophomina sp. in vitro. European Journal of Soil Biology 54: 69-77.

33. Velazquez-Cedeno M, Farnet AM, Mata G, Savoie JM. (2008) Role of Bacillus spp. in antagonism between Pleurotus ostreatus and Trichoderma harzianum in heat-treated wheat-straw substrates. Bioresource Technology 99(15): 6966-6973. 
CC) This work is licensed under Creative

To Submit Your Article Click Here: Submit Article

DOI: 10.32474/CIACR.2018.03.000168

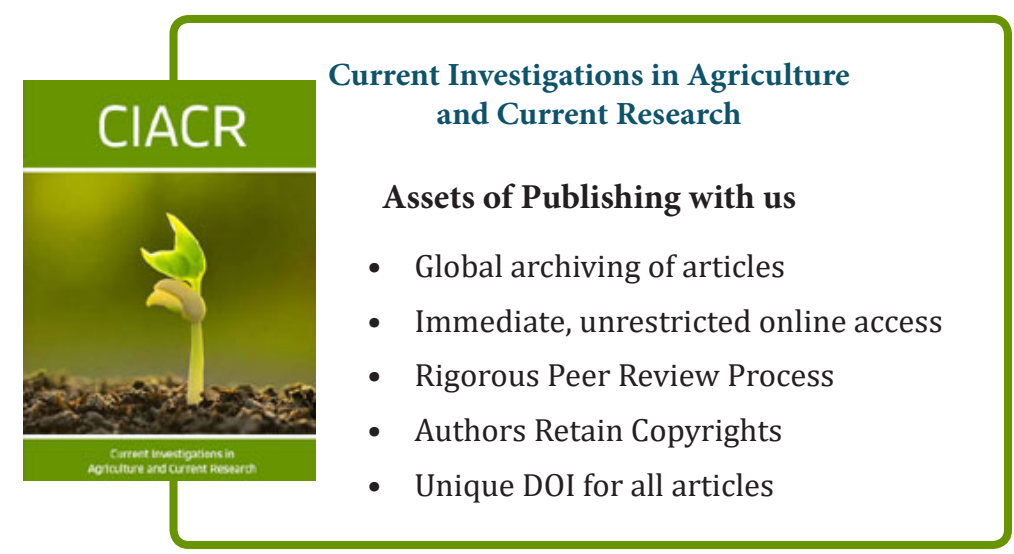

